\author{
Achille CASALE*
}

\title{
Sardostygia (gen. n.) cogonii sp. n., nuovo genere e nuova specie di Coleotteri ipogei di Sardegna \\ (Coleoptera Leiodidae Cholevinae: Leptodirini)
}

\begin{abstract}
Riassunto: Sardostygia (gen. n.) cogonii sp. n., nuovo genere e nuova specie di Coleotteri ipogei di Sardegna (Coleoptera, Leiodidae, Cholevinae: Leptodirini). L'autore descrive una nuova specie di Coleoptera Leptodirini, reperita in una grotta della Sardegna occidentale, altamente modificata e specializzata all'ambiente sotterraneo e appartenente ad un genere inedito: Sardostygia (gen. nov.) cogonii sp. n. Il nuovo taxon è particolarmente distinto da tutte le specie ipogee sarde della linea filetica di Ovobathysciola per numerosi caratteri, quali il tegumento dorsale glabro, il capo privo di carena occipitale, i tarsi anteriori nei maschi non dilatati e l'endofallo privo di strutture sclerificate. Le affinità del nuovo taxon, che in base a un'analisi molecolare risulta affine al genere Ovobathysciola, sono ampiamente discusse.
\end{abstract}

\begin{abstract}
Sardostygia (gen. nov.) cogonii sp. n., new genus and new species of hypogean beetles from Sardinia (Coleoptera, Leiodidae, Cholevinae: Leptodirini).

Sardostygia (gen. nov.) cogonii sp. $n$. is described from a cave of western Sardinia, highly modified and adapted to the subterranean environment. The new taxon is markedly distinct from all other hypogean species known so far from Sardinia of the Ovobathysciola phyletic lineage by many morphological features, as the dorsal integument glabrous, head without occipital carina, fore tarsi in males not dilated and endophallus unarmed, without sclerified copulatory pieces. The relationships of the new taxon, which from a molecular analysis appears to be related to the genus Ovobathysciola, are widely discussed.
\end{abstract}

Key words: Sardostygia gen. nov., cogonii sp. n., Coleoptera, Leiodidae, Cholevinae, Leptodirini, Sardinia, taxonomy, biogeography.

\section{INTRODUZIONE}

Per molti anni (dal 1970 a oggi, per la precisione) mi sono occupato di fauna ipogea di Sardegna. La scoperta recente di una nuova specie di Coleoptera Leptodirini altamente specializzata all'ambiente sotterraneo, attribuibile a un genere inedito, in un massiccio della Sardegna occidentale e in una regione ritenuta ormai ben indagata da un punto di vista biospeleologico (Puddu \& Pirodda, 1974; Cassola, 1982; Grafitti, 2001), mi giunge pertanto come una graditissima e inaspettata sorpresa.

Quattro generi di Leiodidae Cholevinae (= Cholevidae Auctorum) della tribù Leptodirini, tutti includenti specie adattate all'ambiente sotterraneo ed endemiche della Sardegna, sono attualmente noti nell'isola (Perreau, 2000; Casale et al., 2009): Bathysciola Jeannel, 1910, Ovobathysciola Jeannel, 1924, Patriziella Jeannel, 1956 e Batinoscelis Jeannel, 1924, quest'ultimo descritto come sottogenere di Speonomus Jeannel, 1908, ma qui citato come genere distinto.

Tre dei quattro generi sopra menzionati sono stati oggetto recentemente di accurate indagini tassonomiche e biogeografiche (Rampini \& Sbordoni, 1980; Rampini \& Zoia, 1990; Giachino, 1990; Casale, 2004, 2014). I rappresentanti del gen. Bathysciola sono distribuiti in tutta l'isola. Al contrario, le specie ipogee più specializzate dei rimanenti generi erano finora note solo dei massicci calcarei della Sardegna nord-occidentale, centro-orientale e meridionale (Fig. 14).

\section{MATERIALI E METODI}

Le misure e i disegni sono stati ottenuti mediante camere lucide e lenti micrometriche montate su stereomicroscopi Wild M-5 e Wild M-3 e su microscopio composto Leitz Orthoplan. I preparati microscopici, realizzati mediante procedure standardizzate (deidratazione, diafanizzazione), sono montati in Euparal su vetrini allegati ai rispettivi esemplari.

Le fotografie sono state realizzate, con la consueta abilità, da Paolo Magrini (Firenze) mediante camera digitale Nikon D2X applicata su microscopio ottico binoculare Nikon Labophot II o su stereomicroscopio Nikon SMZ 1000, con obiettivi diaframmati.

L'analisi molecolare è stata eseguita nei labora-

${ }^{*}$ Achille Casale, c/o Università di Sassari (Zoologia). Privato: Corso Raffaello 12, 10126 Torino, Italia. E-mail: a_casale@libero.it 
tori dell'IBE di Barcellona. I metodi, i geni e i primer utilizzati nelle sequenze sono quelli indicati da Ribera et al. (2010) e Cieslak et al. (2014a,b).

Le abbreviazioni usate nel testo sono le seguenti:

HT: holotypus;

PT, PTT:paratypus, paratypi;

Misure (minime, massime e medie misurate su

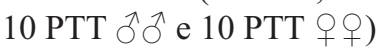

TL: lunghezza totale del corpo, misurata dal margine anteriore del clipeo all'apice delle elitre.

LA: lunghezza delle antenne;

LA/TL: rapporto lunghezza antenne/lunghezza totale del corpo;

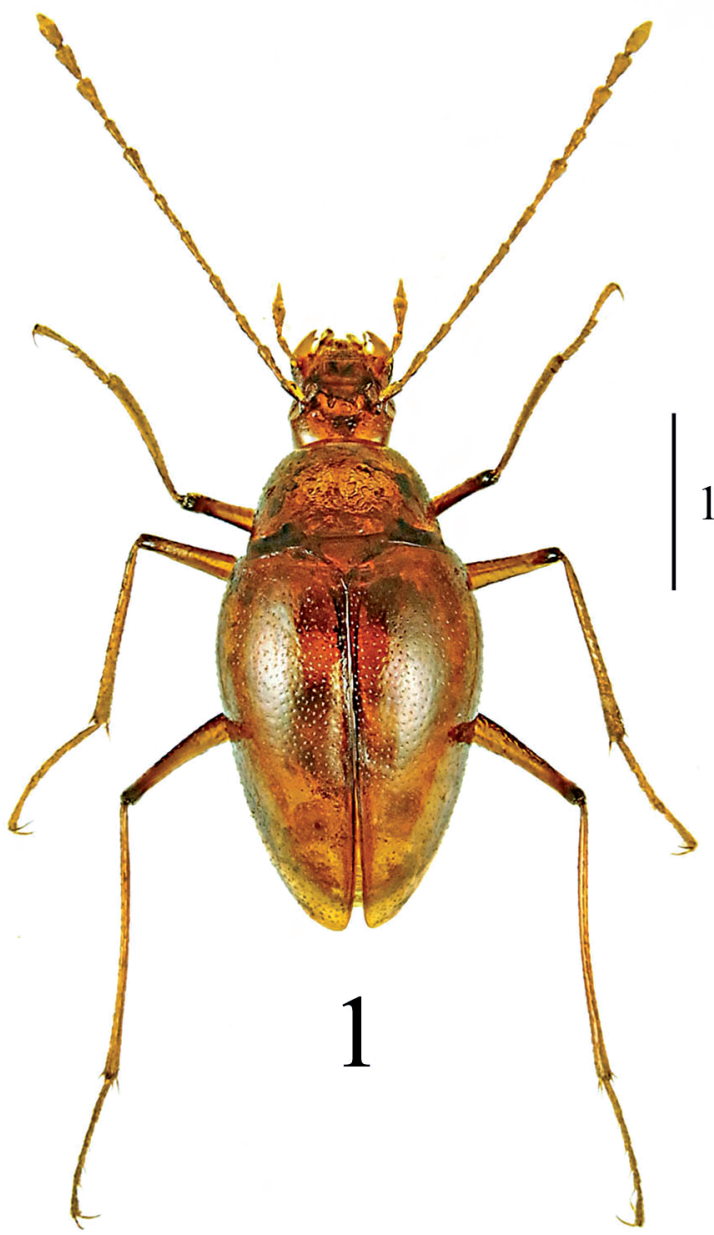

PL/PW: rapporto massima lunghezza del pronoto/ massima larghezza del pronoto;

EL/EW: rapporto lunghezza elitre/massima larghezza elitre;

Acronimi delle collezioni in cui è conservato il materiale studiato:

CCA: coll. dell'Autore (Torino); CFR: coll Javier Fresneda (Museu de Cièncias Naturals, Barcellona, Spagna); CGI: coll. P.M. Giachino (Torino); CGSS: coll. Gruppo Speleologico Sassarese (c/o G. Grafitti, Sassari); CMA: coll. P. Magrini (Firenze); CON: coll. C. Onnis, Maracalagonis (Cagliari); CVA: coll. D. Vailati (Brescia); IBE: Instituto de Biologia Evolutiva, CSIC, Barcellona (Spagna); MSNG: Museo Civico di Storia Naturale G. Doria, Genova.

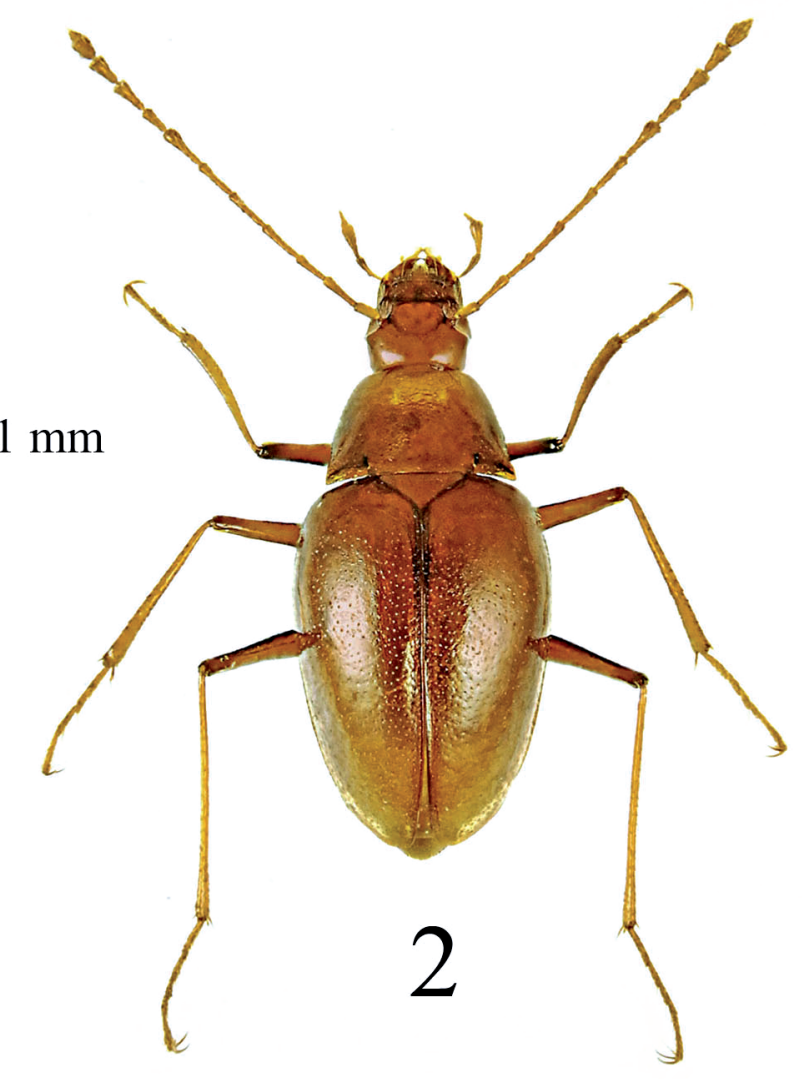

Figg. 1-2. Sardostygia (gen. nov.) cogonii sp. n. 1 - habitus (PT §); 2 - idem (PT †) (foto P. Magrini). 
NotA: i dati catastali della grotta citata nella descrizione, tratti dal Catasto Speleologico della Sardegna, portano la sigla di una delle province "storiche" della Regione: CA: Cagliari; NU: Nuoro; OR: Oristano; SS: Sassari (Fig. 14).

\section{CARATteri del Genere}

Sardostygia gen. nov.

SPECIE TIPO: Sardostygia cogonii sp. nov. (per designazione originale).

DERIVATIO NOMINIS: nome composto dalla patria tipica (Sardegna), e dal suffisso stygia, derivato dal fiume infernale, lo Stige, dell'antica mitologia greca. Per assonanza con un genere di Leptodirini ipogei al medesimo grado di specializzazione troglomorfa, Orostygia G. Müller, 1912, proprio delle Prealpi orientali.

Diagnosi. Genere di Leiodidae Cholevinae Leptodirini nel senso di Newton, 1998 e di Perreau, 2000 (= Cholevidae Leptodirinae nel senso di Giachino et al., 1998; Zoia, 1998; Zoia \& Latella, 2007; Salgado et al., 2008; Casale et al., 2009), caratterizzato dalla combinazione peculiare dei seguenti caratteri morfologici:

Dimensioni medie (TL: $\min : 3.0 \mathrm{~mm}$; max: $3.48 \mathrm{~mm}$; medie: 3,28-3,33 mm). Habitus foleuonoide (Figg. 1 e 2). Tegumenti depigmentati, lucidi, glabri sul lato dorsale, finemente pubescenti sul lato ventrale. Superficie dorsale coperta da punti piccoli, fitti, sparsi, non allineati trasversalmente (Fig. 3).

Capo anoftalmo, privo di carena occipitale; antenne molto allungate, distese all'indietro raggiungenti

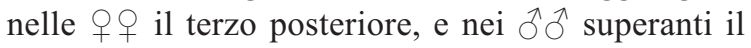
terzo posteriore delle elitre; antennomero 1 distintamente più breve dell'antennomero 2 ; antennomeri 211 tutti distintamente più lunghi che larghi, non formanti una clava antennale. Antennomero 7 dilatato distalmente e con organo di Hamann ben sviluppato. Penultimo articolo dei palpi mascellari 2 volte $e^{1 / 2}$ più lungo dell'ultimo.

Pronoto trasverso, sub-trapezoidale, molto più largo alla base che al margine anteriore, più stretto della massima larghezza delle elitre, con lati non sinuati. Base arcuata e incavata. Angoli anteriori del tutto svaniti, angoli posteriori acuti e molto prominenti.

Scutello molto grande e trasverso. Carena mesosternale molto rilevata (Figg. 10 e 11), con tegumento zigrinato ai lati, ricurva e finemente denticolata sul lato anteriore, non ispessita sul lato ventrale e non prolungata sul metasterno, dove è percettibile un lieve ispessimento appena rilevato.

Mesocoxe e metacoxe separate. Meso- e metaventriti fusi, senza sutura ventrale visibile. Apparato metatergale con apofisi mediana molto sviluppata, raggiungente i 3/4 della lunghezza delle elitre (Fig. 12), simile a quella nota in specie del gen. Patriziella. Metendosternite (organo di Crowson) a Y, con furca molto sviluppata e senza particolari caratteristiche, simile a quello di Ovobathysciola (cfr. Casale, 2014: fig. 26).

Elitre allungate, con massima larghezza al quarto anteriore, attenuate posteriormente, prive di stria suturale.

Zampe molto allungate e gracili (Figg. 4 e 5). Tibie anteriori ricurve, lisce e concave sul lato esterno, prive di spine o di speroni pre-apicali esterni lungo $\mathrm{i}$ margini e prive di corona ("corbeille") o pettine di spine lungo il margine apicale, provviste di 1-2 speroni apicali di dimensioni minori sul lato esterno e di 1 sperone interno di dimensioni maggiori, finemente dentellato. Tarsomeri anteriori nei maschi non dilatati: i sessi sono distinguibili dal numero dei tarsomeri nei protarsi (5 nei maschi, 4 nelle femmine). Unghie tarsali sottili, molto allungate e ricurve.

Segmento genitale maschile senza particolari caratteristiche, come in Ovobathysciola (cfr. Casale, 2014: fig. 27). Ottavo urosternite nelle femmine arrotondato distalmente, con apofisi prossimale breve, larga e subtroncata all'apice (Fig. 13).

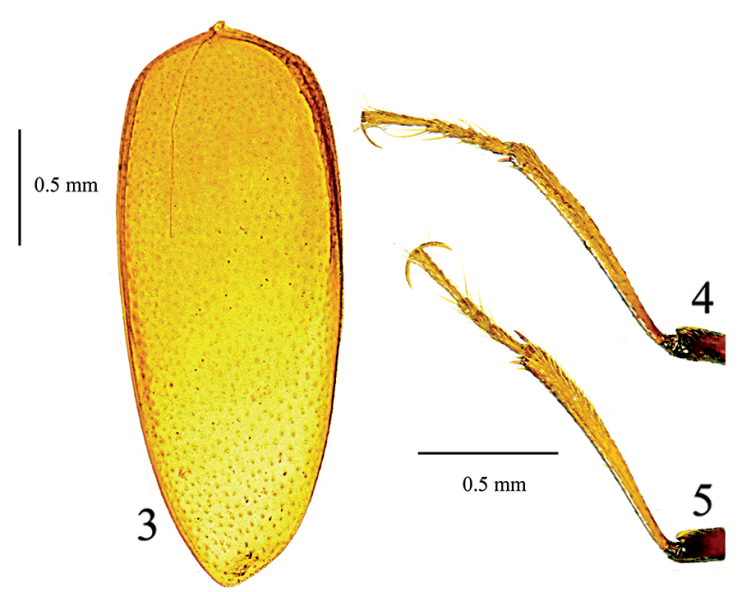

Figg. 3-5. Sardostygia (gen. nov.) cogonii sp. n., caratteri morfologici esterni. 3 - elitra sinistra in visione ventrale (PT †); 4 - zampa anteriore sinistra (PT $\delta$ ) ; 5 - zampa anteriore sinistra (PT $q$ ) (foto P. Magrini). 

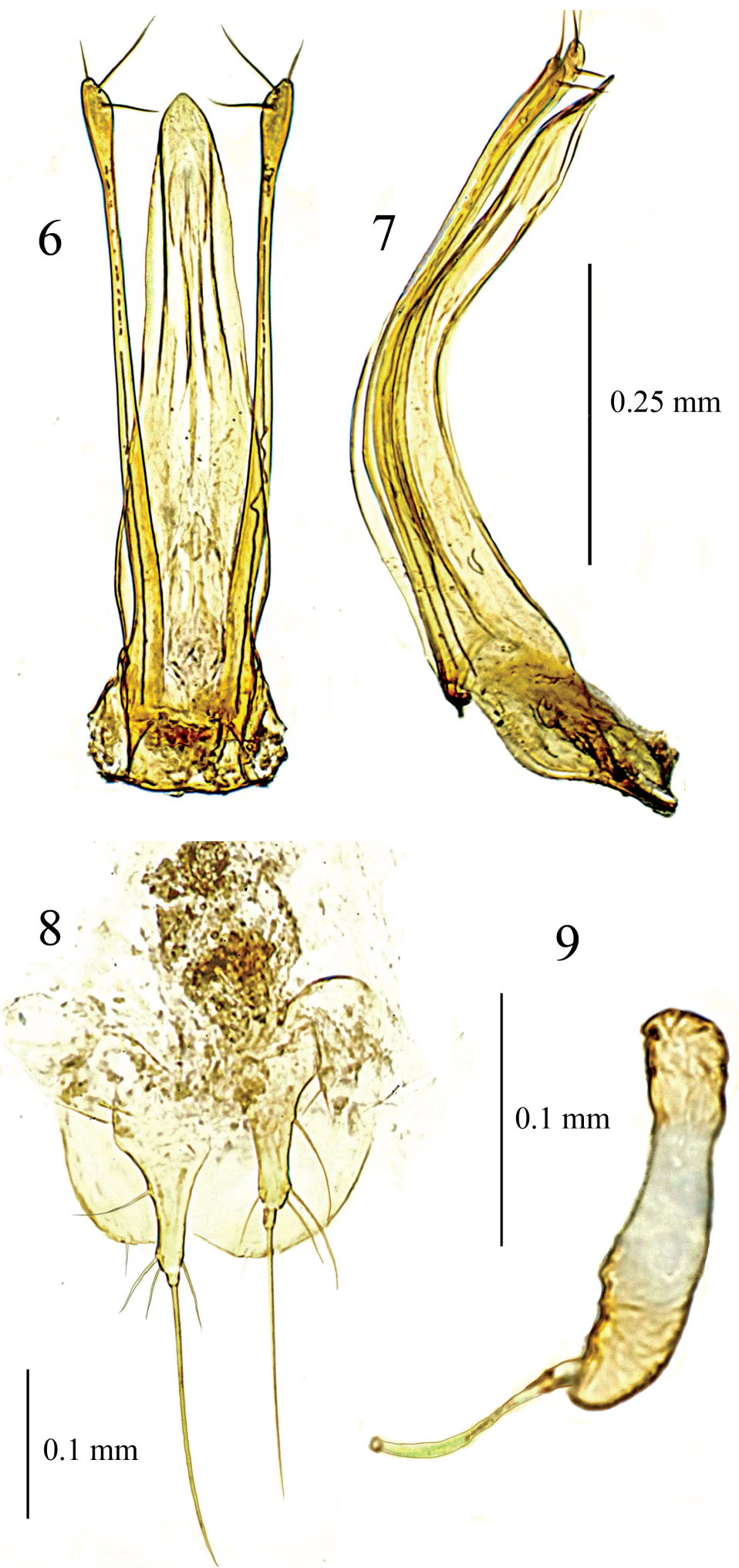

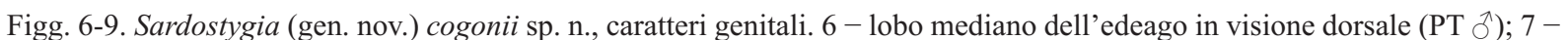
lobo mediano in visione laterale (PT §); 8 - stilomeri dell'ovopositore (PT + ); 9 - spermateca (PT $\circ$ ) (foto P. Magrini). 
Genitali maschili (Figg. 6 e 7): lobo mediano dell' edeago piccolo (lungo $0,58 \mathrm{~mm}$ ), allungato, ricurvo, con regione apicale ben differenziata, sinuosa in visione laterale e regolarmente attenuata, sub-acuminata in visione dorsale e ventrale. Parameri allungati, sinuosi, fortemente ispessiti e claviformi nel tratto distale, superanti l'apice del lobo mediano, ciascuno provvisto di tre setole: due sub-apicali ventrali e una più breve apicale. Endofallo privo di pezzo a $\mathrm{Y}$ basale e di strutture sclerificate differenziate.

Genitali femminili: gonostili piccoli e molto allungati, provvisti di una lunga setola in posizione prebasale, di tre setole preapicali e di una lunghissima setola distale articolata con l'apice del gonostilo (Fig. 8); spermateca di piccolissime dimensioni (lunga 0,13 $\mathrm{mm})$, allungata, molto debolmente sclerificata alla base e all'apice, ialina nel tratto mediano (Fig. 9).

\section{DESCRIZIONE DELLA SPECIE TIPO}

\section{Sardostygia cogonii sp. n. (Figg. 1-13)}

Loc. Typ.: Sardegna (Oristano), Cuglieri (Cavaleddu), Grotta di Turaghile 2944 Sa/OR.

Materiale tiPico: HT $\delta^{\lambda}$ : Sardegna (Oristano), $\mathrm{Cu}-$ glieri, loc. Cavaleddu, 30.VI.2018, R. Cogoni leg. (CCA).

PTT: 31 수우우, stessa località e raccoglitore, 10.II.2018, 30.VI.2018 (CCA, CGI, CGSS, CMA, CON, CVA, MSNG); 1 PT $\partial$, 30.IX.2017 (IBE) (in etanolo assoluto per sequenza di DNA) (IBE); 1 PT , idem (CFR).

DERIVATIO NOMINIS: Dedico con grande piacere questa nuova, bellissima e inattesa specie a Roberto Cogoni, appassionato naturalista e attivo speleologo del Gruppo Speleologico Sassarese, che con la guida di Giuseppe Grafitti ha speso tempo e fatica nelle ricerche che hanno portato alla scoperta del nuovo taxon qui descritto.

DesCrIZIONE: Dimensioni medie: TL: 3,28-3,33 mm (misurate dal margine anteriore del clipeo all'apice delle elitre); minime: 3,0 mm; massime: $3,48 \mathrm{~mm}$.

Corpo convesso, allungato, foleuonoide (Figg. 1 e 2); colore fulvo testaceo uniforme. Tegumenti lucidi, glabri sul lato dorsale, pubescenti sul lato ventrale. Microscultura del pronoto in maglie più evidenti, embricate; punteggiatura delle elitre finissima, sparsa, priva di pubescenza (Fig. 3). Dimorfismo sessuale marcato: nei $\widehat{\partial} \widehat{\jmath}$ le antenne sono nettamente più lunghe in rapporto alla lunghezza del corpo, le elitre sono più strette e allungate nel terzo distale, e i tarsi anteriori sono composti da 5 tarsomeri non dilatati (4 nelle 우우) (Figg. 4 e 5).

Capo allungato, anoftalmo, privo di carena occipitale, che è appena indicata da un ispessimento dorsale fra i lobi laterali del capo. Antenne molto allungate, nei $\widehat{\jmath} \widehat{\lambda}$ pari ai $4 / 5$ della lunghezza del corpo (LA/TL ô $\hat{o}: 0,84 ;$; + ㅇ 0,77 ), più sottili e allungate nei maschi (LA: $\overbrace{}^{\widehat{\jmath}} 2,70 \mathrm{~mm}$, 우 0 2,40 mm), con articoli tutti distintamente più lunghi che larghi, non formanti una "clava" antennale. Antennomero 1 breve, ispessito; antennomero 2 cilindrico, molto allungato, di lunghezza pari a circa il doppio di quella dell'antennomero 1; antennomeri 3-6 molto allungati e gracili, subeguali in lunghezza, circa quattro volte più lunghi che larghi; antennomero 7 di lunghezza pari a quella del 6 , ma dilatato all'apice e con organo di Hamann ben sviluppato; antennomero 8 allungato, di lunghezza pari a circa i $3 / 4$ di quella del 7 ; antennomeri 9 e 10 molto più lunghi che larghi e debolmente dilatati all'apice; antennomero 11 ovoidale allungato, appena più lungo del precedente, ialino all'apice.

Pronoto convesso, molto trasverso, sub-trapezoidale, circa una volta e $1 / 2$ più largo che lungo (PL/PW: 0,50-0,56), con massima larghezza alla base e nettamente più stretto della massima larghezza delle elitre; margini laterali non sinuati, convergenti verso il margine anteriore, divergenti verso il margine posteriore. Margine anteriore appena arcuato; angoli anteriori completamente svaniti. Margine basale arcuato, concavo; angoli basali acuti e prominenti.

Elitre ovalari allungate, convesse, quasi una volta e $1 / 2$ più lunghe che larghe (EL/EW: 1,41-1,45), con massima larghezza anteriormente alla metà della lunghezza, più nettamente ristrette posteriormente nei maschi, con margini laterali lungamente e regolarmente attenuati all'apice. Stria suturale assente, con tracce appena percettibili nel tratto basale e apicale dell'elitra.

Altri caratteri come nella descrizione del genere. Genitali maschili come in Figg. 6 e 7. Genitali femminili come in Figg. 8 e 9.

\section{DISTRIBUZIONE E HABITAT}

I dati che seguono sono stati cortesemente forniti da R. Cogoni e G. Grafitti (Sassari). 
Il taxon qui descritto è noto finora esclusivamente della Grotta di Turaghile 2944 Sa/OR, che si apre in località Cavaleddu nel comune di Cuglieri (Sardegna, Oristano). I dati catastali sono i seguenti: Latitudine N WGS84: 4009'42,80”, Longitudine E WGS84: 8³3'44,60", sviluppo spaziale 159 m, dislivello totale $5 \mathrm{~m}$.

La grotta, recentemente oggetto di nuove prospezioni speleologiche che ne hanno ulteriormente accresciuto lo sviluppo, si apre in vulcaniti trachitico-fonolitiche e basalti di età pliocenica e pleistocenica all'interno di una proprietà privata chiusa da un cancello, ed è costituita da un condotto vulcanico nel quale scorre un ruscello che rende la cavità molto umida, ricca di depositi argillo-limosi con piccole pozze nel fango in cui sono presenti crostacei acquatici.

La temperatura rilevata il 30 giugno 2018 è stata di $14,3^{\circ} \mathrm{C}$, con $81,4 \%$ di umidità.

Le condizioni ambientali all'interno della cavità rappresentano un habitat ideale per la specie qui descritta, che è apparsa abbondante. Gli esemplari della serie tipica sono stati tutti raccolti a vista, senza l'ausilio di esche, vaganti sul suolo umido, su substrato roccioso o sotto pietre, da circa 70 metri dall'ingresso fino alle zone più interne. Sono stati osservati anche numerosi resti di individui morti o predati.

\section{FAUNA ASSOCIATA}

La fauna associata è ricca e comprende numerose specie adattate all'ambiente ipogeo. Sono stati osservati, fotografati e/o campionati nel corso delle ricerche (R. Cogoni, in litteris) i seguenti taxa in studio (determinazioni preliminari di G. Grafitti):

Annelida Hirudinea Gen. sp.

Mollusca Gastropoda Limacidae Limax sp. gruppo corsicus

Mollusca Gastropoda Zonitidae Oxychilus sp.

Arachnida Araneae Gen. sp.

Arachnida Araneae Fam. Tetragnathidae Meta bourneti

Arachnida Araneae Fam. Loxoscelidae?

Arachnida Araneae Fam. Dysderidae?

Arachnida Araneae Fam. Leptonetidae Gen. sp. con occhi ridotti

Crustacea Isopoda Fam. Asellidae (Proasellus sp.?)

Crustacea Isopoda Fam. Stenasellidae (Stenasellus sp.?) anoftalma
Crustacea Isopoda Fam. Trichonisciidae (Catalauniscus sp.?) anoftalma

Insecta, Coleoptera Cryptophagidae Gen. sp.

Diplopoda Fam. Blaniulidae Gen. sp. anoftalma

\section{AFFINITÀ E NOTE SUI CARATTERI MORFOLOGICI}

L'unica specie finora nota del genere qui descritto, Sardostygia (gen. nov.) cogonii sp. n., presenta caratteri morfologici peculiari che la differenziano marcatamente da tutte le specie sarde attribuite ad altri generi. In particolare, nei confronti dei generi con rappresentanti troglomorfi più specializzati (Ovobathysciola e Patriziella), il nuovo taxon è nettamente distinto per la combinazione dei seguenti caratteri: 1, tegumenti dorsali glabri; 2, capo privo di carena occipitale; 3 , endofallo privo di strutture sclerificate. Alcuni caratteri coincidono con quelli del genere iberico Spelaeochlamys Dieck, 1870 (Salgado et al., 2008), all'interno del quale tuttavia le specie (come in tutte

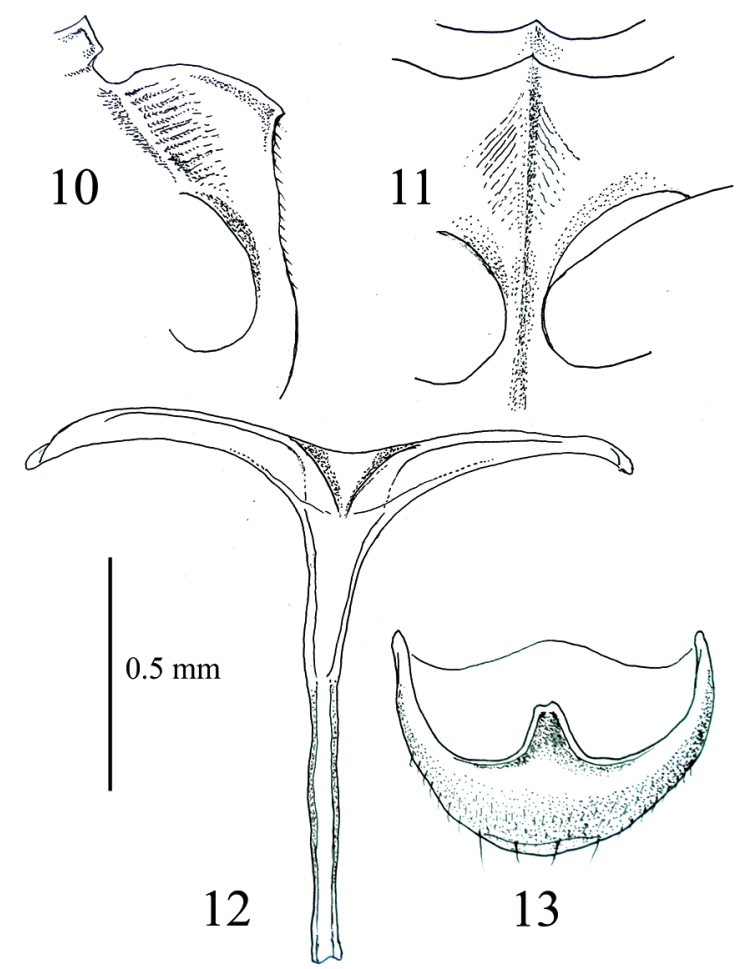

Figg. 10-13. Sardostygia (gen. nov.) cogonii sp. n., caratteri morfologici esterni. 10 - carena mesosternale in visione laterale (PT đ) ; 11 - carena mesosternale in visione ventrale (PT đ̂); 12 - apparato metatergale (PT +); 13 - ottavo segmento genitale femminile in visione ventrale (PT $q$ ). 
quelle della "serie di Spelaeochlamys") sono pubescenti e presentano l'endofallo munito di strutture sclerificate basali e/o apicali.

Merita tuttavia ricordare che fra i Leptodirini sardi Bathysciola fortesculpta Jeannel, 1914, endemica del massiccio del Monte Albo, presenta tegumenti dorsali glabri. La posizione sistematica di questa specie non è stata ancora chiarita: marcatamente isolata da tutte le altre specie sarde di Bathysciola (Giachino, 1990), essa presenta tuttavia un'armatura dell'endofallo che la allontana morfologicamente anche da tutte le specie della linea filetica di Ovobathysciola (cfr. Casale, 2014, fig. 71).

Jeannel (1956) ha evidenziato il valore informativo dei caratteri dell'endofallo che si riscontrano in tutte le specie della "linea filetica di Ovobathysciola": in particolare, la presenza di una peculiare fanera mediana a $\mathrm{T}$ o ad "ancora rovesciata", accompagnata dall'assenza di una fanera a Y basale. Nel nuovo taxon qui descritto l'endofallo risulta ialino e inerme.

Un'analisi molecolare di Sardostygia (n. gen.) cogonii n. sp., condotta su un esemplare paratipo (vedi materiale tipico) nei laboratori dell'IBE di Barcellona, dove è disponibile un grande database di sequenze di Leptodirini di tutta l'area euro-mediterranea, ha rivelato con un buon supporto che il nuovo taxon è in realtà un elemento molto derivato all'interno del complesso di Ovobathysciola-Patriziella, pur permanendo ancora incertezze sulle relazioni fra le varie specie all'interno del gruppo (Ribera, in litteris).

La corretta individuazione della posizione sistematica di alcuni generi e specie di Leptodirini presenti in Sardegna è problematica ed è stata oggetto di numerose ipotesi. Come ricordato in una sintesi recente $(\mathrm{Ca}-$ sale, 2014), per quanto riguarda le affinità che legano i due generi Ovobathysciola e Patriziella (nel senso degli autori) e i livelli di adattamento (grado di troglomorfismo) che presentano le singole specie, si rimanda a Rampini \& Sbordoni (1980) e Rampini \& Zoia (1990).

Nelle varie classificazioni proposte, tali generi compaiono in posizioni molto differenti: cfr. Jeannel (1911, 1924, 1956), Laneyrie (1967), Guéorguiev (1976), Casale et al. (1991), Newton (1998), Giachino et al. (1998), Salgado \& Fresneda (2004), Fresneda et al. (2007, che evidenziano un clade includente la "serie di Spelaeochlamys" e la "serie di Ovobathysciola"), Salgado et al. (2008). Nel catalogo di Perreau (2000), Ovobathysciola e Patriziella sono inserite in una "série phylétique de Ovobathysciola", nella sottotribù Pholeuina di Leptodirini.

Caccone \& Sbordoni (2001) hanno esaminato sia i tempi di speciazione fra i taxa specifici conosciuti, sia la monofilia e la separazione dei due generi di Sardegna attribuiti alla serie filetica di Ovobathysciola. Nel medesimo contributo tali autori, su dati molecolari tarati su basi geologiche e paleo-ambientali, $\mathrm{o}$ a dati più generali sui tassi di evoluzione in organismi ipogei (Sbordoni et al., 1999), hanno proposto uno scenario della colonizzazione dell'ambiente sotterraneo e dei tempi di divergenza e di speciazione delle specie oggi attribuite a Ovobathysciola, rispetto a quanto ipotizzato per le specie afferenti a Patriziella.

Successivamente Casale $(2004,2014)$ ha sostenuto la monofilia di un gruppo formato dalle tre specie attribuite a Patriziella, accomunate da caratteri sinapomorfi, non adattativi, che escludono l'ipotesi di ridurre Ovobathysciola e Patriziella al rango di generi "di convenienza" (Rampini \& Zoia, 1990). I dati morfologici e biogeografici avvalorano infatti la separazione generica fra le specie attribuite a Ovobathysciola dalle tre specie note attribuite a Patriziella, queste ultime confinate in singole cavità ad andamento verticale, profonde e fredde, sovrapposte e inserite nell'areale di Ovobathysciola (Patriziella nuragica Rampini \& Zoia, 1990 è parapatrica rispetto a $O$. grafittii, mentre P. sardoa Jeannel, 1956 e P. muceddai Casale, 2004 sono sintopiche con O. majori, e P. muceddai pure con $O$. supramontana) (Fig. 14).

Si ritiene verosimile datare ora, su basi molecolari, l'origine dei Leptodirini a circa $44 \mathrm{Ma}$ e l'origine dei Leptodirini mediterranei al tardo Eocene-Oligocene (42 $\pm 5 \mathrm{Ma}$ ) (Ribera et al., 2010; Fresneda et al., 2011; Cieslak et al., 2014), calibrata sui tempi del distacco della placca sardo-corsa (33 Ma) nel quadro dell'evoluzione del Paleo-Mediterraneo occidentale (Alvarez, 1972; Boccaletti et al., 1990; Casale \& Vigna Taglianti, 1996; Cassola, 1982; Cherchi \& Montadert, 1982; Hsü et al., 1977; Steininger \& Rögl, 1984; Popov et al., 2004; Fresneda et al., 2007; Ribera et al., 2010).

Per future indagini, occorrerà considerare anche le linee di popolamento, di dispersione (anche in territori non calcarei) e di isolamento allopatrico che hanno interessato le numerose popolazioni di Leptodirini frazionate in Sardegna, su un territorio interessato da estrema complessità geologica e geo-morfologica e da profonde e alterne modificazioni climatiche nel corso del Plio-Pleistocene, che hanno 
profondamente mutato la copertura vegetazionale dell'area (La Greca, 1990, 1998). La morfologia e la distribuzione del taxon qui descritto aggiungono pertanto nuovi dati sui rappresentanti ipogei di Coleop- tera Cholevinae presenti nell'isola, testimoni attuali di progenitori miocenici già adattati alla vita in ambiente sotterraneo che non hanno lasciato tracce in linee epigee attuali (Ribera et al., 2010).

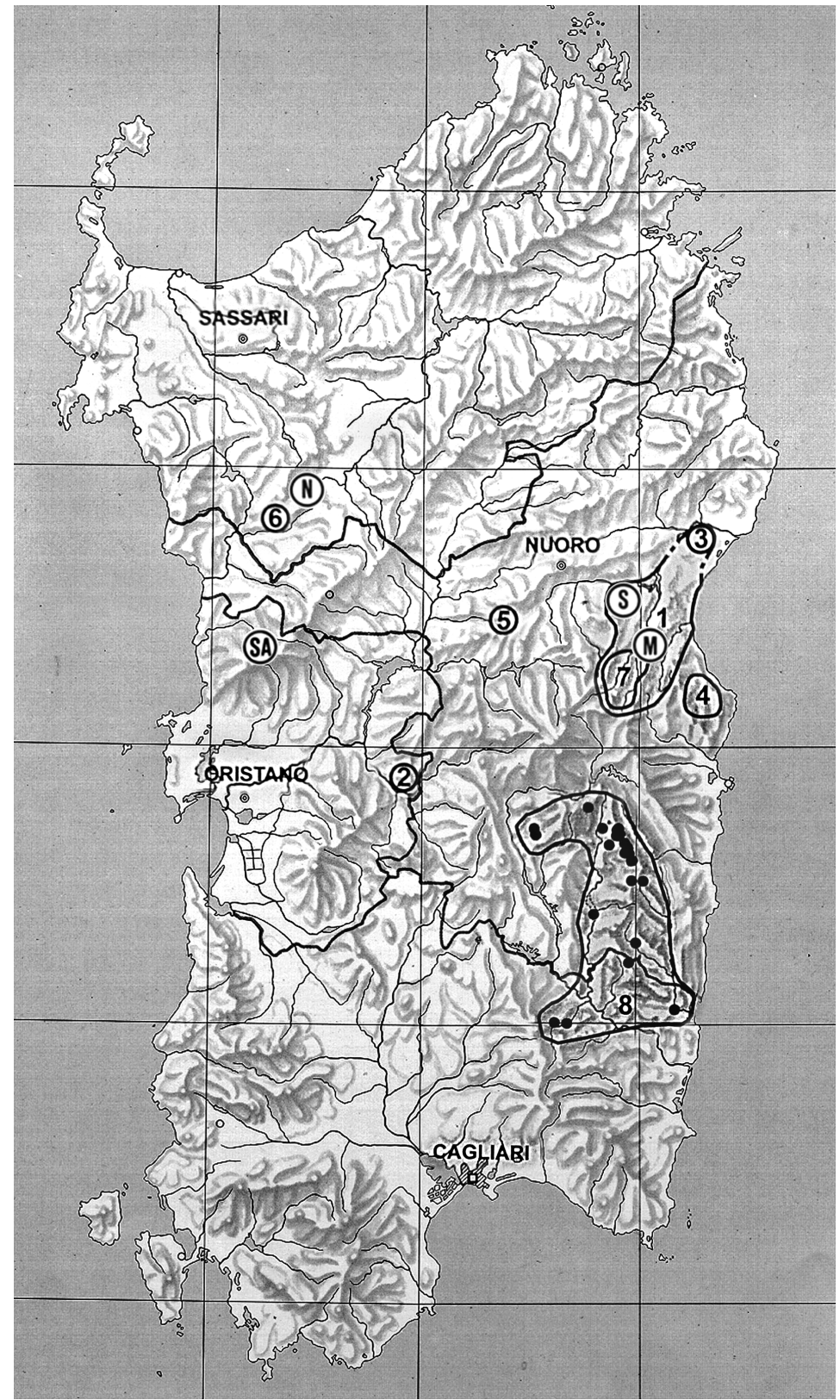

Fig. 14. Distribuzione nota delle specie della linea filetica di Ovobathysciola in Sardegna (modificata da Casale, 2014). 1: Ovobathysciola majori (Reitter, 1885); 2: O. castrimedusae Casale, 2014; 3: O. deleddae Casale, 2014; 4: O. paeoniae Casale, 2014; 5: O. magrinii Casale, 2014; 6: O. grafittii Rampini \& Sbordoni, 1980; 7: O. supramontana Casale, 2014; 8: O. gestroi (Fairmaire, 1872); M: Patriziella muceddai Casale, 2004; N: P. nuragica Rampini \& Zoia, 2014; S: P. sardoa Jeannel, 1956; SA: Sardostygia (gen. nov.) cogonii sp. n. 


\section{RINGRAZIAMENTI}

Per avermi fornito il materiale su cui è basata la presente descrizione, sono molto grato agli amici del Gruppo Speleologico Sassarese Roberto Cogoni, appassionato esploratore di cavità e attento raccoglitore di campioni biospeleologici, e Giuseppe Grafitti, da decenni infaticabile animatore e coordinatore della Biospeleologia sarda.

Un ringraziamento particolare è inoltre rivolto all'amico Paolo Magrini (Firenze), che con la consueta maestria ha eseguito le macrofotografie e ha composto le tavole che illustrano la presente descrizione.

Ringrazio inoltre vivamente l'Assessorato alla Difesa dell'Ambiente della Regione Autonoma della Sardegna (Maria Ledda, Direttore del Servizio) per l'autorizzazione concessa a Roberto Cogoni per i campionamenti nella grotta di Su Turaghile, i colleghi e amici Pier Mauro Giachino e Dante Vailati per la lettura critica del presente manoscritto, e l'amico Ignacio Ribera dell'IBE di Barcellona per l'analisi molecolare del nuovo taxon, con preziosi commenti sulla sua posizione tassonomica.

\section{BIBLIOGRAFIA}

AlVAREZ W., 1972 - Rotation of the Corsica-Sardinia microplate. Nature, London, 235: 103-105.

BocCaletti M., Ciaranfi N., CASentino D., 1990 - Palinspastic restoration and paleogeographic reconstruction of the peri-Tyrrhenian area during the Neogene. Palaeogeography, Palaeoclimatology, Palaeoecology, Elsevier, Amsterdam, 77: 41-50.

CAcCone A., SBordoni V., 2001 - Molecular biogeography of cave life: a study using mitochondrial DNA from bathysciine beetles. Evolution, Raleigh, 55(1): 122-130.

CASALE A., 2004 - Due nuovi Coleotteri ipogei di Sardegna, Sardaphaenops adelphus n. sp. (Coleoptera Carabidae) e Patriziella muceddai n. sp. (Coleoptera Cholevidae), e loro significato biogeografico. Bollettino della Società entomologica italiana 136(1): 3-31.

CASALE A., 2014 - Il genere Ovobathysciola Jeannel, 1924, endemico di Sardegna, con descrizione di cinque nuove specie (Coleoptera, Cholevidae, Leptodirini). Annali del Museo civico di Storia naturale "G. Doria", 106: 223-289.

Casale A., Giachino P. M., Vailati D., 1991 - Brevi considerazioni per una sistematica filogenetica dei Bathysciinae (Coleoptera: Cholevidae). Atti XVI Congresso nazionale italiano di Entomologia, Bari - Martina Franca (TA): 857-865.

Casale A., Grafitti G., Latella L., 2009 - The Cholevidae (Coleoptera) of Sardinia (pp. 290-316). In: P. Cerretti, F. Mason, A. Minelli, G. Nardi \& D. Whitmore (eds.), Research on the Terrestrial Arthropods of Sardinia (Italy). Zootaxa, Auckland, 2318: 1-602.

Casale A., Vigna Taglianti A., 1996 - Coleotteri Carabidi di Sardegna e delle piccole isole circumsarde, e loro significato biogeografico (Coleoptera, Carabidae). Biogeographia, Lavori della Società italiana di Biogeografia, Siena, (n.s.), 18(1995): 391-427.

CASsola F., 1982 - Il popolamento cavernicolo della Sardegna. Lavori della Società italiana di Biogeografia, Siena, (n.s.), 7 (1978): 615-755.

CHERCHI A., MONTADERT L., 1982 - Oligo-Miocene rift of Sardinia and the early history of the western Mediterranean basin. Nature, London, 298: 736-739.

Cieslak A, Fresneda J, Ribera I., 2014a - Developmental constraints in cave beetles. Biology Letters, 10: 20140712.

Cieslak A, Fresneda J, Ribera I., 2014b - Life-history specialization was not an evolutionary dead-end in Pyrenean cave beetles. Proceedings of the Royal Society B, 281: 20132978.

Fresneda J., Salgado J.M., Ribera I., 2007 - Phylogeny of western Mediterranean Leptodirini, with an emphasis on genital characters (Coleoptera: Leiodidae: Cholevinae). Systematic Entomology, London, 32: 332-358.

Fresneda J., GrebenNiKov V.V., Ribera I., 2011 - The phylogenetic and geographic limits of Leptodirini (Insecta: Coleoptera: Leiodidae: Cholevinae), with a description of Sciaphyes shestakovi sp. n. from the Russian Far East. Arthropod Systematics \& Phylogeny, 69: 99-123.

Giachino P. M., 1990 - Note sulle Bathysciola di Sardegna. Sistematica, corologia e zoogoeografia delle specie affini a $B$. damryi (Abeille, 1881) (Col., Cholevidae, Bathysciinae). Annali del Museo civico di Storia naturale "G. Doria", Genova, 88: 301-329.

Giachino P. M., VAilati D., CASAle A., 1998 - Major questions in the phylogeny and biogeography of Cholevidae (Coleoptera), with emphasis on the subfamily Leptodirinae (pp. 179-210). In: GiaChINo P.M. \& PECK S. (eds.), Phylogeny and Evolution of Subterranean and Endogean Cholevidae (= Leiodidae Cholevinae). Museo Regionale di Scienze Naturali, Torino, Atti (Proceedings of XX I.C.E., Firenze, 1996), 296 pp. 
Grafitti G., 2001 - Osservazioni sulla fauna cavernicola della Sardegna (pp. 13-33). In: Piras, G. \& Randaccio, F. (eds.), Atti Convegno "Biospeleologia dei sistemi carsici della Sardegna" (Cagliari, 10 giugno 2000). Gruppo speleologico Centro Studi ipogei "Specus", Cagliari, 116 pp.

GuÉorguiev V.B., 1976 - Recherches sur la taxonomie, la classification et la phylogénie des Bathysciinae (Coleoptera: Catopidae). Slovenska Akademija Znanosti in Umetnosti, Razprave Dissertationes, Ljubljana, 19/4: 92-147.

Hsü K.J., Montadert L., Bernoulli D., CitaM. B., Erickson A., Garrison R.E., Kidd R.B., Melieres F., Müller C., Wright R., 1977 - History of the Mediterranean Salinity Crisis. Nature, London, 267: 399-403.

JEANNEL R., 1911 - Biospeologica. XIX. Révision des Bathysciinae (Coléoptères Silphides). Morphologie, Distribution géographique, Systematique. Archives de Zoologie expérimentale et générale, Paris 7(5): 1-641.

Jeannel R., 1924 - L. Biospeologica. Monographie des Bathysciinae. Archives de Zoologie expérimentale et générale, Paris 63(1): 1-436.

JEANNEL R., 1956 - Sur un Bathysciite cavernicole nouveau de la Sardaigne (Coleoptera Catopidae). Fragmenta entomologica, 2(10): 105-114.

LA GreCA M., 1990 - The insect biogeography of west Mediterranean islands. Atti Convegno Lincei "Biogeographical aspects of insularity", Roma (1987): 459-468.

LA GReCA M., 1998 - La situazione paleoclimatica nel Quaternario. Biogeographia, Siena, 19: 1-29.

LANEYRIE R., 1967 - Nouvelle classification des Bathysciinae (Coléoptères Catopidae). Tableaux des sous tribus, groupes de genres et genres. Annales de Spéléologie, Moulis, 22(3): 585-645.

Newton A. F., 1998 - Phylogenetic problems, current classification and generic catalogue of world Leiodidae (including Cholevidae). In: Giachino P.M. \& PECK S. (eds.), Phylogeny and Evolution of Subterranean and Endogean Cholevidae (= Leiodidae Cholevinae). Museo Regionale di Scienze Naturali, Torino, Atti (Proceedings of XX I.C.E., Firenze, 1996), pp. 41-178.

Perreau M., 2000 - Catalogue des Coléoptères Leiodidae Cholevinae et Platypsyllinae. Mémoires de la Société entomologique de France, Paris, 4: 1-460.

Popov S.V., Rögl F., Rozanov A.Y., Steininger F.F., Shcherba I.G., Kovac M. (eds.), 2004 - Lithological-Paleogeographic maps of Paratethys. 10 Maps late Eocene to Pliocene. Courier Forschungsinstitut Senckenberg, Frankfurt am Main, 250: 1-46, 10 maps.

Puddu S., Pirodda G., 1974 - Catalogo sistematico ragionato della fauna cavernicola della Sardegna. Rendiconti dei Seminari della Facoltà di Scienze, Università di Cagliari, 73 (3-4) (1973): 151-205.

Rampini M., Sbordoni V., 1980 - Una nuova Ovobathysciola di Sardegna (Coleoptera, Catopidae). Fragmenta entomologica, 15(2): 337-344.

Rampini M., Zoia S., 1990 - Un nuovo Batiscino cavernicolo di Sardegna: Patriziella nuragica n. sp. (Coleoptera, Cholevidae). Fragmenta entomologica, 22(2): 369-380.

Ribera I., Fresneda J., Bucur R., Izquierdo A., Vogler A., Salgado J., Cieslak A., 2010 - Ancient origin of a Western Mediterranean radiation of subterranean beetles. BMC Evolutionary Biology, 10: 29.

Salgado J.M., Fresneda J., 2004 - Revision of the section Anillochlamys Jeannel, 1909 (Coleoptera: Leiodidae: Cholevinae: Leptodirini). Annales de la Société entomologique de France, Paris, (n.s.), 39(4) (2003): 361-384.

Salgado J.M., Blas E. M., Fresneda J.G., 2008 - Fauna Iberica. 31. Coleoptera Cholevidae - CSIC, Madrid, 787 pp.

Sbordoni V., Allegrucci G., Cesaroni D., 1999 - Population genetic structure, speciation and evolutionary rates in cave dwelling organisms (pp. 219-240). In: Wilken H., Culver D.C. \& Humphreys W.F. (eds.), Subterranean ecosystems. Elsevier, Amsterdam, $791 \mathrm{pp}$.

Steininger F.F., Rögl F., 1984 - Paleogeography and palinspastic reconstruction of the Neogene of the Mediterranean and Paratethys (pp. 659-668). In: DiXON J.E. \& RoBERTSON A.H.F. (eds.), The geological evolution of the eastern Mediterranean. Geological Society, London, $836 \mathrm{pp}$.

ZoIA S., 1998 - Considerations of the present knowledge of the Italian Cholevidae and their distribution, with particular reference to the hypogean species (Coleoptera) (pp. 211-226). In: Giachino P.M. \& PECK S. (eds.), Phylogeny and Evolution of Subterranean and Endogean Cholevidae (= Leiodidae Cholevinae). Museo regionale di Scienze naturali, Torino, Atti (Proceedings of XX I.C.E., Firenze, 1996), 296 pp.

Zoia S., Latella L., 2007 - Insecta Coleoptera Cholevidae and Platypsyllidae (pp. 177-180). In: Ruffo S. \& Stoch F. (eds.), Checklist and distribution of the Italian fauna. Memorie del Museo civico di Storia naturale, Verona, 2a ser., Sez. Scienze della Vita, 17 (2006), 304 pp. (maps in CD rom). 